\title{
Alojamento Conjunto, Amamentação e Seguimento Neonatal de Recém-Nascidos de Mãe com COVID-19
}

\author{
Rooming-in, Breastfeeding and Neonatal Follow-up of \\ Infants Born to Mothers with COVID-19
}

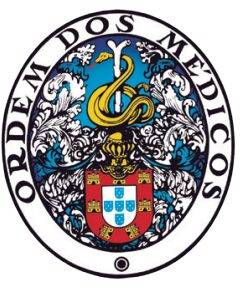

Isabel BRITO ${ }^{1}$, Rita SOUSA 1 , Bruno SANCHES ${ }^{1,2}$, João FRANCO ${ }^{1,2}$, Susana MARCELINO ${ }^{1,2}$, Anselmo COSTA ${ }^{1,2}$ Acta Med Port 2021 Jul-Aug;34(7-8):507-516 - https://doi.org/10.20344/amp.15441

\section{RESUMO}

Introdução: Dada a evidência crescente de maior benignidade da COVID-19 no recém-nascido, surgiram recomendações de promoção do alojamento conjunto e da amamentação. O principal objetivo do estudo foi avaliar a segurança dessa abordagem, através do risco de infeção neonatal grave.

Material e Métodos: Estudo observacional prospetivo de abril 2020 a fevereiro 2021 da abordagem hospitalar e seguimento após a alta dos recém-nascidos de mãe com COVID-19 num hospital com apoio perinatal diferenciado, onde foram advogados o alojamento conjunto e amamentação, sempre que possível. Recolhemos os dados no internamento e em seguimento telefónico durante o período neonatal.

Resultados: Incluímos 77 recém-nascidos de mãe com COVID-19 (3,8\% do total de recém-nascidos), com medianas de idade gestacional 39 semanas e 5 dias e $2370 \mathrm{~g}$ de peso à nascença; destes, $9 \%$ nasceram pré-termos (versus $12 \%$ pré-termos no total de recém-nascidos). Todos estiveram em alojamento conjunto e $4 \%$ foram admitidos transitoriamente na Unidade de Cuidados Intensivos Neonatais; um total de $88 \%$ recém-nascidos tiveram alta até ao terceiro dia de vida, $97 \%$ tiveram alta sob aleitamento materno e 90\% mantinham-no no fim do período neonatal. Dos 63 recém-nascidos com seguimento telefónico completo, oito tiveram sintomas compatíveis com COVID-19, três dos quais com observação médica. Em 40\% dos casos não houve consulta médica de vigilância após a alta. Houve 5\% recém-nascidos com COVID-19 (num total de quatro, registámos um quadro ligeiro e três assintomáticos), sem particularidades no internamento ou seguimento.

Discussão: A infeção neonatal foi incomum, não houve quadros graves nem maior incidência de prematuridade. O alojamento conjunto e a amamentação foram práticas seguras, devendo ser promovidas desde que clinicamente possível. Destacamos que a vigilância de saúde após a alta necessita de ser melhorada.

Conclusão: Os recém-nascidos de mãe com COVID-19 podem ser mantidos em alojamento conjunto e sob aleitamento materno exclusivo.

Palavras-chave: Alojamento Conjunto; Amamentação; COVID-19; Portugal; Recém-Nascido; SARS-CoV-2

\section{ABSTRACT}

Introduction: Due to growing evidence suggesting COVID-19 may have a benign course in the newborn, a number of guidelines supporting rooming-in and breastfeeding were developed. The main aim of the study was to assess the safety of this approach, through the risk of developing severe neonatal infection.

Material and Methods: Prospective observational study from April 2020 to February 2021 on the approach and neonatal follow-up of infants born to mothers with COVID-19 at the time of delivery in a hospital with advanced neonatal care, where rooming in and breastfeeding were promoted whenever possible. We collected data during hospital admission and over the phone during the neonatal period Results: We included 77 infants born to mothers with COVID-19 (3.8\% of newborns born during the time of study), median gestational age 39 weeks +5 days and median birth weight 3270 g; $9 \%$ were born premature (versus 12\% born premature among newborns born during the time of study). Rooming-in took place in all of them although $4 \%$ were briefly admitted to the Neonatal Intensive Care Unit; $88 \%$ were discharged home up to day three, $97 \%$ were breastfed at the time of discharge and $90 \%$ were still breastfed by the end of the neonatal period. We completed neonatal follow-up of 63 newborns, eight of them developed COVID-associated symptoms, three with need of medical evaluation; $40 \%$ had no medical assessment after being discharged. Out of $77,5 \%$ of infants were infected with SARS-CoV-2 (total of four, one mild, three asymptomatic), with no significant differences during hospital stay or follow-up.

Discussion: Neonatal infection was uncommon and mild, and there was no increase in prematurity. Rooming-in and breastfeeding were safe and should be promoted whenever clinically possible. Follow-up care after hospital discharge needs improvement.

Conclusion: Infants born to mothers with COVID-19 were safely roomed in with their mothers and exclusively breastfed.

Keywords: Breast Feeding; COVID-19; Infant, Newborn; Portugal; Rooming-in Care; SARS-CoV-2

\section{INTRODUÇÃO}

A infeção por severe acute respiratory syndrome coronavirus 2 (SARS-CoV-2) foi reportada pela China à Organização Mundial da Saúde a 31 dezembro $2019^{1}$ e declarada como pandemia a 11 março de $2020 .^{2}$

A doença provocada por SARS-CoV-2 (COVID-19) é uma infeção respiratória aguda, de clínica variável, com dois terços dos infetados a apresentarem sintomas ${ }^{3}-$ entre esses, a maioria $(81 \%)$ apresenta doença ligeira e $5 \%$ doença crítica. ${ }^{4}$ Há menor incidência e menor gravidade na idade pediátrica, incluindo no período neonatal. . $^{5-8}$

No recém-nascido $(\mathrm{RN})$, a doença é incomum e o principal foco de transmissão é a mãe. ${ }^{9,10}$ Após a introdução da

1. Serviço de Pediatria. Hospital Garcia de Orta. Almada. Portugal.

2. Unidade de Cuidados Intensivos Neonatais. Hospital Garcia de Orta. Almada. Portugal.

$\triangle$ Autor correspondente: Isabel Brito. isabelmarrbrito@gmail.com

Recebido: 13 de fevereiro de 2021 - Aceite: 30 de abril de 2021 - Online issue published: 01 de julho de 2021 Copyright $\odot$ Ordem dos Médicos 2021 
pesquisa universal de SARS-CoV-2 na parturiente durante a admissão hospitalar, a evidência sugere que até $15 \%$ das grávidas tem COVID-19 e até $90 \%$ dessas são assintomáticas. ${ }^{11}$ Os respetivos $\mathrm{RN}$ podem ter um risco aumentado de infeção, ${ }^{12}$ o que motivou uma ampla discussão sobre a abordagem à díade mãe-RN. ${ }^{10,13}$

Inicialmente, as recomendações das principais sociedades de Pediatria e Neonatologia seguiram duas correntes distintas: a corrente chinesa, que recomendava a separação mãe-filho e aleitamento adaptado exclusivo, ${ }^{14}$ e a corrente da Sociedade Italiana de Neonatologia (SIN), que recomendava alojamento conjunto e amamentação com medidas de prevenção de transmissão. ${ }^{15}$ As recomendações da Sociedade Portuguesa de Neonatologia (SPN) ${ }^{16}$ seguiram a linha italiana, enquanto a American Academy of Pediatrics (AAP) ${ }^{10}$ e o Royal College of Paediatrics and Child Health $(\mathrm{RCPCH})^{17}$ recomendavam a separação mãe-filho e evicção da amamentação.

O principal objetivo deste estudo foi avaliar a segurança do alojamento conjunto do RN de mãe com COVID-19, através da avaliação do risco de infeção neonatal grave. Os objetivos secundários foram caracterizar a população de RN de mãe com COVID-19 em termos demográficos, epidemiológicos e de variáveis perinatais como prematuridade, transmissibilidade e espectro da infeção neonatal por SARS-CoV-2, evolução clínica, bem como caracterizar a vigilância em Cuidados de Saúde Primários (CSP) prevista no Programa Nacional de Saúde Infantil e Juvenil (PNSIJ). ${ }^{18}$

\section{MATERIAL E MÉTODOS}

Este estudo observacional prospetivo decorreu entre 1 de abril de 2020 e 07 de fevereiro de 2021 no Hospital Garcia de Orta, um hospital de nível II com apoio perinatal diferenciado, onde vigora um plano de contingência COVID-19 específico para a díade mãe-RN, adotado desde abril 2020, e onde a recolha de dados se mantém em curso. Esse plano inclui: decisão partilhada com a mãe sobre alojamento conjunto em isolamento e amamentação, desde que a condição clínica de ambos permita; impossibilidade de visitas durante o internamento; explicação de dúvidas e preocupações da mãe; critérios e cuidados habituais respeitantes à via do parto, clampagem tardia do cordão, contacto pele a pele, administração de vitamina K e profilaxia ocular; medidas de prevenção de transmissão em todos os momentos incluindo uso de máscara, higienização das mãos, lavagem do peito e abdómen da mãe, berço a dois metros da cama da mãe no alojamento conjunto, uso de equipamento de proteção individual; pesquisa de SARS-CoV-2 no RN; alta com a mãe pelos critérios clínicos habituais; e seguimento telefónico uma ou duas vezes por semana durante os primeiros 28 dias de vida do RN, complementando o seguimento presencial nos CSP.

De acordo com o Plano, os cuidados perinatais dos RN de mãe com COVID-19 seriam o mais semelhantes possível aos dos de RN de mãe sem COVID-19, com a promoção do alojamento conjunto (o internamento da mãe e do RN no mesmo espaço físico, com o berço junto à cama da mãe), com os mesmos critérios para decisão da via do parto, admissão na unidade de Cuidados Intensivos Neonatais $(\mathrm{UCIN})$ e alta. As principais diferenças seriam o internamento numa enfermaria de isolamento, as medidas de prevenção de transmissão, a pesquisa de SARS-CoV-2 no $\mathrm{RN}$, a realização do rastreio metabólico antes do terceiro dia de vida (com parecer favorável do Instituto Nacional de Saúde Doutor Ricardo Jorge) e a realização do rastreio auditivo neonatal universal (RANU) após a alta hospitalar.

A população-alvo do estudo incluiu todos os RN de mãe com COVID-19 que cumprissem cumulativamente quatro critérios:

1) Parto hospitalar ou parto não-hospitalar com assistência pós-parto no hospital do estudo;

2) Mãe com infeção ativa por SARS-COV-2, definida por deteção de SARS-CoV-2 no exsudado naso/orofaríngeo em pesquisa por real time polymerase chain reaction (RT-PCR) na admissão hospitalar ou por diagnóstico prévio sem conclusão do protocolo de cura;

3) Condição clínica compatível com a opção de alojamento conjunto, definida pela estabilidade clínica do RN e da mãe. Impossibilitariam o alojamento conjunto, por exemplo, a presença de síndrome de dificuldade respiratória (SDR) precoce, a prematuridade (menos de 33 semanas) ou dificuldade alimentar mantida - que seriam critérios para admissão do RN em UCIN. De modo similar, condições maternas como pré-eclampsia grave ou patologia respiratória (COVID-19 ou não) com necessidade de internamento em $\mathrm{UCl}$, também impediram o alojamento conjunto.

4) Mãe concordante com a inclusão no estudo.

Excluímos qualquer RN cuja mãe tivesse pesquisa de SARS-CoV-2 positiva à admissão hospitalar, mas em quem não fosse assumida infeção ativa ou que revogasse o consentimento informado em qualquer momento do seguimento.

Recolhemos dados relativos a: enquadramento epidemiológico intrafamiliar - contactos, diagnóstico e clínica da puérpera e coabitantes; variáveis perinatais - via do parto, idade gestacional, peso à nascença, necessidade de reanimação e de internamento em UCIN; abordagem e evolução clínica a nível hospitalar - pesquisa de SARS-CoV-2 no RN, decisão, tipo e condições de alojamento, alimentação do RN, duração do internamento e critérios para alta; e seguimento após a alta hospitalar - evolução clínica do $\mathrm{RN}$, da mãe e dos coabitantes, vigilância epidemiológica e protocolo de cura da mãe e do RN (quando aplicável), vigilância em CSP e cuidados gerais do RN.

Obtivemos os dados nas observações clínicas do RN em internamento, através da consulta dos processos clínicos e do seguimento telefónico durante o período neonatal - feito uma ou duas vezes por semana, com obtenção de informação relativa ao estado clínico do RN e dos seus coabitantes (incluindo a mãe), aos cuidados gerais do RN, à amamentação, ao acompanhamento pelos CSP e a quaisquer preocupações parentais. Os dados foram armazenados em Microsoft Exce ${ }^{\circledR}$ para posterior análise 
estatística descritiva simples.

O estudo foi aprovado pela Comissão de Ética do Hospital Garcia de Orta.

Foi pedido consentimento informado das parturientes.

\section{RESULTADOS}

Os resultados são referentes ao período entre 1 de abril e 31 de dezembro de 2020.

\section{1) Abordagem hospitalar}

Nasceram 2124 RN no hospital, de 2074 puérperas. Houve 3,8\% (78/2074) puérperas infetadas por SARS-CoV-2 no momento do parto e 3,7\% (78/2124) RN de mães com COVID-19 - 77 incluídos no presente estudo, um excluído pela ausência de consentimento materno (Fig. 1).

\section{a) Puérperas (Tabela 1)}

As puérperas com COVID-19 tinham uma mediana de 28 anos (mínimo 18, máximo 42 anos). Das 77, 83\% (64/77) teve o diagnóstico no rastreio à admissão e $17 \%$ (13/77) tinham diagnóstico prévio, sem terem ainda sido consideradas curadas; destas, 12 tinham tido infeção sintomática - uma com necessidade de internamento seis semanas antes do parto por pneumonia a SARS-CoV-2.

Apenas $10 \%$ das puérperas (8/77) apresentava sintomas (ligeiros) no momento do parto. As restantes $90 \%$ $(69 / 77)$ eram assintomáticas. Houve $3 \%$ puérperas $(2 / 77)$
2124 nados-vivos

durante 9 meses no hospital de estudo
78 recém-nascidos de mãe com COVID-19
Excluídos:

1 por recusa materna de inclusão no estudo

77 recém-nascidos incluídos

63 recém-nascidos de mãe com COVID-19 com seguimento telefónico durante o período neonatal completo

Seguimentos perdidos:

- 11 por contactos telefónicos incorretos;

- 2 por ausência de resposta após um primeiro contacto;

- 1 por internamento em alojamento conjunto durante todo o período previsto de seguimento (causa materna - pneumonia a SARS-CoV-2).

Figura 1 - Fluxograma da elegibilidade para o estudo 
Tabela 1 - Puérperas com COVID-19 $(n=77)$

\begin{tabular}{lrrr}
\hline Idade & & & anos \\
& Mediana & & 28 \\
Mínima & & 18 \\
Diagnóstico & Máxima & & 42 \\
& & $\mathbf{n}$ & \% \\
& Prévio & 64 & $83 \%$ \\
Clínica & À admissão & 13 & $17 \%$ \\
& & 77 & $100 \%$ \\
& & $\mathbf{n}$ & \% \\
& & 58 & $75 \%$ \\
& Ligsintomática & 16 & $21 \%$ \\
& Grave & 3 & $4 \%$ \\
& & 77 & $100 \%$ \\
\hline
\end{tabular}

com necessidade de prolongamento do internamento por pneumonia a SARS-CoV-2.

b) Parto e pós-natal imediato (Tabela 2)

Dos 77 RN, 52\% (40/77) eram do sexo feminino e 55\% (42/77) nasceram por parto eutócico. Dos partos distócicos (35), 74\% (26/35) foram por cesariana: por estado fetal não tranquilizador $(35 \%, 9 / 26)$, falha na progressão do trabalho de parto $(23 \%, 6 / 26)$, antecedentes maternos de cesariana $(27 \%, 7 / 26)$, patologia materna $(12 \%, 3 / 26)$ e incompatibilidade feto-pélvica $(4 \%, 1 / 26)$.

A idade gestacional variou entre 33 semanas e quatro dias e 41 semanas e dois dias, (mediana 39 semanas e cinco dias). Houve 9\% (7/77) RN pré-termos entre os RN de mãe com COVID-19; entre RN de mãe sem COVID-19, houve $12 \%$ pré-termos (246/2046).

$\mathrm{O}$ peso à nascença variou entre $2030 \mathrm{~g}$ e $4150 \mathrm{~g}$ (mediana $3270 \mathrm{~g}$ ), sendo a maioria dos $\mathrm{RN}$ adequados à idade gestacional [AIG (91\%, 70/77)]; identificaram-se 6\% (5/77) de RN leves para a idade gestacional (LIG) e 3\% (2/77) grandes para a idade gestacional.

Em 5\% (4/77) dos casos houve necessidade de manobras de reanimação neonatal; destes, apenas um tinha um índice de Apgar inferior a 7 ao quinto minuto. Três tiveram rápida recuperação e ficaram internados em alojamento conjunto com a mãe. O outro RN também recuperou rapidamente, mas foi admitido na UCIN durante a estabilização obstétrica da mãe, ficando de seguida em alojamento conjunto até à alta. Não houve necessidade de reanimação neonatal de nenhum RN pré-termo.

Todos os RN reuniram condições clínicas para alojamento conjunto durante pelo menos parte do internamento. Em 4\% (3/77) houve necessidade de admissão transitória na UCIN: dois diretamente do bloco de partos (um por SDR precoce e o outro, como já referido, pela condição materna) e um admitido por dificuldade respiratória e alimentar em D10 vida (ainda internado na altura por motivos sociais). A este último foi diagnosticada uma cardiopatia congénita associada a taquicardia supraventricular paroxística, com
Tabela 2 - Recém-nascido de mãe com COVID-19: parto e pós-natal imediato $(n=77)$

\begin{tabular}{|c|c|c|}
\hline Sexo & $\mathbf{n}$ & $\%$ \\
\hline Feminino & 40 & $52 \%$ \\
\hline Via de parto & $\mathbf{n}$ & $\%$ \\
\hline Eutócico & 42 & $55 \%$ \\
\hline Fórceps & 4 & $5 \%$ \\
\hline Ventosa & 5 & $6 \%$ \\
\hline \multirow[t]{2}{*}{ Cesariana } & 26 & $34 \%$ \\
\hline & 77 & $100 \%$ \\
\hline Idade gestacional & \multicolumn{2}{|c|}{ semanas + dias } \\
\hline \multirow[t]{2}{*}{ Mediana (mín. - máx.) } & \multicolumn{2}{|c|}{$39+5(33+4-41+2)$} \\
\hline & $\mathbf{n}$ & $\%$ \\
\hline Pré-termo, < 37s & 7 & $9 \%$ \\
\hline Termo, [37 - 42s[ & 70 & $91 \%$ \\
\hline \multirow[t]{2}{*}{ Pós-termo, $\geq 42 \mathrm{~s}$} & 0 & $0 \%$ \\
\hline & 77 & $100 \%$ \\
\hline \multicolumn{2}{|l|}{ Peso à nascença } & $\mathbf{g}$ \\
\hline \multirow[t]{2}{*}{ Mediana (mín. - máx.) } & \multicolumn{2}{|c|}{$3270(2030-4150)$} \\
\hline & $\mathbf{n}$ & $\%$ \\
\hline AIG & 70 & $91 \%$ \\
\hline LIG & 5 & $6 \%$ \\
\hline GIG & 2 & $3 \%$ \\
\hline
\end{tabular}

consequente insuficiência cardíaca congestiva; as pesquisas de SARS-COV-2 foram negativas. O RN admitido por SDR precoce era pré-termo, melhorou nas primeiras 24 horas e foi transferido para alojamento conjunto até à alta ao terceiro dia de vida; as pesquisas de SARS-CoV-2 foram negativas. Não houve necessidade de admissão em UCIN de nenhum dos outros RN pré-termos.

c) Alojamento conjunto, amamentação e cuidados perinatais (Tabela 3 )

Por decisão partilhada entre a mãe e a equipa médica, $100 \%$ (77/77) das díades mãe/RN estiveram em alojamento conjunto, em isolamento com medidas de prevenção de transmissão. Os três RN admitidos na UCIN foram os únicos que não acompanharam a mãe durante a totalidade do internamento.

Dos 77 RN, 52\% (40/77) estiveram sob aleitamento materno exclusivo, 3\% (2/77) ficaram sob aleitamento artificial exclusivo (um por opção materna, um por mãe com infeção por vírus da imunodeficiência humana) e os restantes $45 \%$ (35/77) tiveram alta sob aleitamento misto. Houve dois RN (um pré-termo e um LIG) com episódios de hipoglicémia.

No internamento, foi administrada a primeira dose da vacina contra o vírus da hepatite $\mathrm{B}$ à totalidade dos $\mathrm{RN}$; a $94 \%(72 / 77)$ foi feito o rastreio de doenças metabólicas e a $5 \%$ (4/77) foi feito o rastreio auditivo neonatal universal.

A mediana de internamento foi dois dias (mínimo um, máximo 36$)$ e $88 \%(68 / 77)$ teve alta até ao terceiro dia de vida. Houve oito internamentos com mais de três dias por 
causa materna ou social (incluindo dois internamentos por pneumonia a SARS-CoV-2 na mãe) e um por cardiopatia congénita, sendo este o único RN que não teve alta com a mãe (transferência para outro hospital).

\section{d) Pesquisa de SARS-CoV-2 (Tabela 3)}

Em 97\% (75/77) dos RN foi colhido exsudado naso/ orofaríngeo para pesquisa de SARS-CoV-2 por RT-PCR; destes, em $81 \%(61 / 75)$ foram realizadas duas pesquisas (às 24 e 48 horas de vida). A diferença deveu-se à introdução da norma 026/202026 de 19 de maio de 2020 da Direção Geral da Saúde (DGS), a partir da qual transitámos de uma pesquisa única às 24 horas para as duas pesquisas realizadas na maioria dos $\mathrm{RN}$ - a segunda pesquisa foi realizada nos casos em que a primeira foi negativa e foi dispensada nos casos em que a primeira foi positiva. Em quatro casos, correspondendo a $5 \%$ dos RN de mãe com COVID-19, obtiveram-se pesquisas de SARS-CoV-2 positivas: dois nas primeiras 24 horas e dois com pesquisa inicial negativa e positiva às 48 horas.

Neste subgrupo de quatro RN com pesquisa de SARS-CoV-2 positiva, nasceram todos AIG, um RN pré-termo (36 semanas e seis dias) e os restantes três de termo. Nenhum necessitou de reanimação neonatal ou admissão em UCIN. Ficaram em alojamento conjunto com a mãe durante todo o internamento (mediana dois dias) e tiveram alta sob aleitamento materno exclusivo $(50 \%, 2 / 4)$ ou misto $(50 \%, 2 / 4)$. Nenhum dos RN com pesquisa de SARS-CoV-2 positiva desenvolveu infeção sintomática durante o internamento. Das mães, uma tinha sintomas ligeiros e as restantes estavam assintomáticas.

Tabela 3 - Recém-nascidos de mãe com COVID-19: alojamento conjunto $(n=77)$

\begin{tabular}{|c|c|c|}
\hline \multicolumn{2}{|l|}{ Duração de internamento } & dias \\
\hline \multirow[t]{2}{*}{ Mediana (mín. - máx.) } & & $(1-36)$ \\
\hline & $\mathbf{n}$ & $\%$ \\
\hline$\leq 3$ dias & 68 & $88 \%$ \\
\hline \multirow[t]{2}{*}{ Alojamento conjunto } & $\mathbf{n}$ & $\%$ \\
\hline & 77 & $100 \%$ \\
\hline \multirow[t]{2}{*}{ Admissão em UCIN } & $\mathbf{n}$ & $\%$ \\
\hline & 3 & $4 \%$ \\
\hline Amamentação & $\mathbf{n}$ & $\%$ \\
\hline Materna exclusiva & 40 & $52 \%$ \\
\hline Mista & 35 & $45 \%$ \\
\hline Artificial exclusiva & 2 & $3 \%$ \\
\hline Cuidados perinatais gerais & $\mathbf{n}$ & $\%$ \\
\hline Vacina anti-VHB & 77 & $100 \%$ \\
\hline Rastreio metabólico & 72 & $94 \%$ \\
\hline RANU & 4 & $5 \%$ \\
\hline Pesquisa de SARS-CoV-2 no RN & $\mathbf{n}$ & $\%$ \\
\hline$\geq 1$ & 75 & $97 \%$ \\
\hline$>1$ & 61 & $79 \%$ \\
\hline Positiva & 4 & $5 \%$ \\
\hline
\end{tabular}

2) Seguimento durante o período neonatal (Tabela 4)

Dos 77 RN de mãe com COVID-19 nascidos em 2020, concluímos o seguimento telefónico durante pelo menos os primeiros 28 dias de vida em 82\% (63/77). Para manter o apoio às famílias que tínhamos como propósito, mantivemos o seguimento telefónico além do período neonatal nos casos em que no momento da alta prevista (28 dias) ainda não tinha havido início do acompanhamento médico pelos CSP. Dos 18\% (14/77) de casos sem seguimento completo, este foi impossibilitado por ausência de contacto telefónico (11/14), ausência de resposta após o primeiro contacto (2/14) e internamento materno por pneumonia a SARS-CoV-2 durante todo o período neonatal (1/14) (Fig.1). No caso RN com cardiopatia congénita, o seguimento telefónico foi mantido com o hospital para o qual foi transferido e com a mãe. Nenhum dos RN sem seguimento telefónico foi admitido na Urgência Pediátrica do hospital do estudo durante o período neonatal.

\section{a) Contexto epidemiológico intrafamiliar}

Houve três casos de famílias monoparentais. Na maioria dos restantes núcleos familiares $(58 \%, 43 / 74)$ não foi feita pesquisa de SARS-CoV-2 nos coabitantes. Nos 31 núcleos familiares em que foi feita testagem, houve $42 \%$ $(13 / 31)$ em que um ou mais coabitantes tinha COVID-19 e em $23 \%$ desses (3/13) pelo menos um coabitante tinha idade pediátrica.

Durante o seguimento, 97\% (61/63) das mães foram consideradas curadas, $43 \%$ (26/61) sem realização de novo teste; cinco mães mantiveram ou desenvolveram sintomas ligeiros após a alta, mantendo-se as restantes assintomáticas.

Tabela 4 - Recém-nascido de mãe com COVID-19: seguimento telefónico até ao $28^{\circ}$ dia de vida $(n=77)$

\begin{tabular}{rrr}
\hline Seguimentos perdidos & $\mathbf{n}$ & $\%$ \\
Contacto incorreto & 11 & $14 \%$ \\
Ausência de resposta mantida & 2 & $3 \%$ \\
Internamento hospitalar $\geq 28$ dias & 1 & $1 \%$ \\
Seguimentos completos (n = 63) & 14 & $18 \%$ \\
Consulta CSP $\leq 14$ dias & $\mathbf{n}$ & $\%$ \\
Consulta CSP $\leq 28$ dias & 36 & $11 \%$ \\
Avaliação ponderal & 53 & $84 \%$ \\
Ganho ponderal adequado & 48 & $76 \%$ \\
Amamentação ao 28 dia & & \\
Materna exclusiva & 35 & $56 \%$ \\
Mista & 22 & $35 \%$ \\
Artificial exclusiva & 6 & $10 \%$ \\
Sintomas compatíveis com CoVID-19 & $\mathbf{8}$ & $\mathbf{1 3 \%}$ \\
Obstrução nasal & 6 & $10 \%$ \\
Noção dificuldade respiratória & 2 & $3 \%$ \\
Motivo para observação médica & 3 & $5 \%$ \\
\hline
\end{tabular}




\section{b) Evolução clínica}

Durante o seguimento, 13\% (8/63) dos RN desenvolveram sintomas compatíveis com COVID-19: seis com obstrução nasal e dois com noção materna de dificuldade respiratória. Em cinco dos seis casos de obstrução nasal foi ajustada a frequência da vigilância telefónica para apoio parental. O outro RN com obstrução nasal foi observado na Urgência Pediátrica e teve alta clinicamente bem, tal como um dos RN cuja mãe tinha uma noção não confirmada de dificuldade respiratória. O outro $\mathrm{RN}$ com alegada dificuldade respiratória foi observado em visita domiciliária e a sua observação era normal. Não lhes foi repetida a pesquisa de SARS-CoV-2, que poderia ser positiva, dada a coexistência de sintomatologia e contexto epidemiológico.

\section{c) Cuidados gerais do RN}

Dos 63 RN em quem concluímos seguimento, $52 \%$ (33/63) tinham tido alta sob aleitamento materno e 56\% (35/63) RN estavam sob aleitamento materno exclusivo ao $28^{\circ}$ dia de vida - os restantes sete, entre os 40 que tinham tido alta sob aleitamento exclusivo, estão incluídos nos seguimentos perdidos. Ao $28^{\circ}$ dia de vida, $35 \%$ (22/63) encontravam-se sob aleitamento misto e $10 \%$ (6/63) sob aleitamento artificial. Dos 52\% (33/63) que tinham tido alta sob aleitamento materno exclusivo, oito transitaram para aleitamento misto; dos $44 \%$ (28/63) que tinham tido alta sob aleitamento misto, dez transitaram para aleitamento materno exclusivo. Em 84\% (53/63) houve avaliação da evolução ponderal, considerada inadequada em 9\% (5/53).

Os cinco RN a quem não tinha sido feito o rastreio metabólico foram submetidos ao mesmo até ao sexto dia de vida, em visita domiciliária ou consulta de enfermagem dos CSP.

Os 73 RN a quem não tinha sido feito o RANU em internamento foram convocados pelo serviço de Otorrinolaringologia até aos dois meses de vida, exceto num caso rastreado aos cinco meses, como confirmámos em consulta posterior dos registos informáticos.

As principais preocupações parentais foram referentes a cólica do lactente (5), icterícia (4) e amamentação/evolução ponderal (3). Houve uma manifestação informal de satisfação com o seguimento telefónico.

\section{d) Observações médicas}

Dos RN com seguimento telefónico completo, 57\% (36/63) tiveram consulta nos CSP no período neonatal e $17 \%(11 / 63)$ nas primeiras duas semanas de vida. Houve dois RN observados na primeira semana de vida em visita domiciliária pela equipa hospitalar do seguimento telefónico, por decisão médica face à ausência de resposta dos CSP: num caso por noção não confirmada de dificuldade respiratória e noutro para consulta de vigilância de $\mathrm{RN}$ com COVID-19. Um destes RN teve consulta nos CSP ao $14^{\circ}$ dia de vida. Outro RN manteve o internamento noutro hospital durante o restante período neonatal para estabilização em serviço de Cardiologia Pediátrica. Os restantes $40 \%$ (25/63) não tiveram observação médica após a alta.
Tabela 5 - Recém-nascidos com COVID-19 $(n=4)$

\begin{tabular}{|c|c|c|}
\hline Duração internamento & & dias \\
\hline \multirow[t]{2}{*}{ Mediana (mín. - máx.) } & & $(2-3)$ \\
\hline & $\mathbf{n}$ & $\%$ \\
\hline Termo & 3 & $75 \%$ \\
\hline AIG & 4 & $100 \%$ \\
\hline Reanimação neonatal & 0 & $0 \%$ \\
\hline Admissão UCIN & 0 & $0 \%$ \\
\hline \multicolumn{3}{|l|}{ Diagnóstico COVID-19 } \\
\hline$<24 h$ & 2 & $50 \%$ \\
\hline $24-48 h$ & 2 & $50 \%$ \\
\hline Notificação no SINAVE & 3 & $75 \%$ \\
\hline Sintomas compatíveis com COVID-19 & 1 & $25 \%$ \\
\hline Cura & 4 & $100 \%$ \\
\hline Com teste & 1 & $25 \%$ \\
\hline Consulta CSP $\leq 14$ dias & 1 & $25 \%$ \\
\hline Consulta CSP $\leq 28$ dias & 3 & $75 \%$ \\
\hline Observação médica $\leq 28$ dias & 4 & $100 \%$ \\
\hline Avaliação ponderal & 4 & $100 \%$ \\
\hline Ganho ponderal adequado & 4 & $100 \%$ \\
\hline \multicolumn{3}{|l|}{ Amamentação à alta } \\
\hline Materna exclusiva & 2 & $50 \%$ \\
\hline Mista & 2 & $50 \%$ \\
\hline Artificial exclusiva & 0 & $0 \%$ \\
\hline \multicolumn{3}{|l|}{ Amamentação ao $28^{\circ}$ dia } \\
\hline Materna exclusiva & 3 & $75 \%$ \\
\hline Mista & 0 & $0 \%$ \\
\hline Artificial exclusiva & 1 & $25 \%$ \\
\hline
\end{tabular}

\section{e) RN com COVID-19 (Tabela 5)}

Para vigilância epidemiológica, fizemos a notificação no SINAVE em três dos quatro casos. Nenhum dos RN teve seguimento dirigido em Trace-COVID e foram todos considerados curados durante o período neonatal pela equipa de seguimento da mãe, com teste confirmatório da cura em um dos quatro casos.

Destes quatro RN, um desenvolveu obstrução nasal ligeira ao $21^{\circ}$ dia de vida, com boa evolução clínica. Os restantes mantiveram-se assintomáticos.

Dos dois que tiveram alta sob aleitamento misto, um transitou para aleitamento materno exclusivo e um para aleitamento artificial por noção materna de hipogalactia. Os dois que tinham tido alta sob aleitamento materno exclusivo mantiveram-no. Ambos tiveram evoluções ponderais adequadas.

Os quatro RN com COVID-19 foram observados no período neonatal: três em consulta nos CSP, um deles na segunda semana de vida; ao outro RN foi feita visita domiciliária na primeira semana de vida para consulta de vigilância. 


\section{DISCUSSÃO}

\section{1) Dificuldades e limitações do estudo}

A implementação do Plano de Contingência exigiu reorganização logística para permitir o alojamento conjunto em isolamento, com uma exigente articulação entre serviços, com medidas de prevenção de transmissão e segurança para os profissionais de saúde, sem compromisso da humanização dos cuidados. Apesar do esforço, a diminuição do tempo de contacto da mãe com os profissionais pode ter condicionado menor apoio em áreas como a amamentação, contribuindo para que $45 \%$ tenha tido alta sob aleitamento misto, um valor mais alto do que o reportado no último relatório do Observatório do Aleitamento Materno ${ }^{20}$ de 2013, nos Hospitais Amigos dos Bebés em Portugal onde se indicava que $79 \%$ dos RN tinha alta sob aleitamento materno exclusivo e apenas $19 \%$ sob aleitamento misto.

Em relação à falha no seguimento após a alta hospitalar pode dever-se às limitações impostas pelo isolamento ou pela quebra geral na atividade assistencial, mas a ausência de um grupo de controlo de RN de mãe sem COVID-19 limita as conclusões que podemos tirar a esse respeito.

A generalização dos resultados do estudo está limitada pelo reduzido tamanho da população e também pela perda de quase um quinto da população durante o seguimento telefónico.

\section{2) Abordagem hospitalar}

Construímos a abordagem adotada a partir das recomendações da SIN e da SPN. ${ }^{15,16}$ Apesar de fundamentada, a estratégia escolhida foi inovadora já que, no início da pandemia, organizações como a AAP, a RCPCH e a DGS recomendavam abordagens em linha com as orientações chinesas. $10,14,17,19$

No panorama nacional, ${ }^{21}$ durante abril e maio, $61 \%$ dos serviços/unidades de Neonatologia alojavam o RN separado da mãe e $70 \%$ sem aleitamento materno. Para a alta do RN, $43 \%$ exigiam um cuidador com pesquisa de SARS-CoV-2 negativa e $24 \%$ também a cura da mãe, o que prolongou os internamentos, o afastamento de mãe-filho e a evicção da amamentação. ${ }^{22-24} \mathrm{~A}$ opção de separação mãe-filho e evicção da amamentação começou precocemente a ser questionada, porque não assegurava a prevenção da transmissão (sobretudo após a alta), comprometia os benefícios do contacto pele a pele para o RN e o sucesso da amamentação, podia comprometer a estabilidade emocional da mãe e exigia um maior esforço logístico hospitalar, que poderia não ser comportável nos momentos mais exigentes da pandemia. ${ }^{25}$

Desde o princípio da pandemia, promovemos o alojamento conjunto, que o estudo confirma ser seguro, e assistimos à evolução das abordagens em outras unidades nesta direção. 10,17,26 Tivemos adesão ao alojamento conjunto por $100 \%$ das mães e à amamentação em $97 \%$ dos casos. Parto, admissão em UCIN e alta hospitalar foram decididos pelos critérios clínicos habituais. Todos os recém-nascidos foram considerados e abordados como casos suspeitos de COVID-19, mas com cuidados perinatais o mais próximo possível dos habituais.

No global, à semelhança de outro estudo realizado em Itália, ${ }^{13}$ estes resultados apoiam o predomínio dos benefícios sobre os riscos no alojamento conjunto de RN de mãe com COVID-19. Apesar de uma incidência de COVID-19 nas parturientes inferior à descrita no estudo publicado no New England Journal of Medicine ${ }^{1}{ }^{1}(3,8 \%$ vs $15 \%)$, a proporção de infeção assintomática foi semelhante $(90 \%$ vs $88 \%$ ). Contrariamente a outras revisões em que até $13 \%$ grávidas tinham doença grave, ${ }^{27}$ no presente estudo não houve doença materna crítica, e apenas uma grávida e duas puérperas tiveram doença grave (pneumonia a SARS-CoV-2 com necessidade de internamento). Segundo a revisão do The BMJ sobre COVID-19 na gravidez, ${ }^{27} 25 \%$ dos recém-nascidos de mãe com COVID-19 são admitidos na UCIN, o que não verificámos neste estudo (4\%). É de salientar que na revisão não estão explícitos os motivos de internamento e a opção de separação mãe-filho pode justificar uma maior taxa de admissão em UCIN sem implicar existência de critérios clínicos de admissão.

A taxa de necessidade de reanimação neonatal foi mais baixa do que o descrito em trabalhos anteriores $(5 \%$ vs $13 \%),{ }^{12}$ à semelhança do verificado em relação à prematuridade - $9 \%$ vs $23 \%-45 \%$ 12,28,29; por outro lado, não houve maior taxa de prematuridade nos RN de mãe com COVID-19 comparativamente com a população geral no mesmo período ( $9 \%$ vs $12 \%$ ). Não houve óbitos na população em estudo; a nível mundial, apesar de terem sido descritos casos raros de mortalidade em RN com COVID-19, nenhum viu essa causalidade confirmada. ${ }^{13,29}$

\section{3) Seguimento no período neonatal}

O seguimento telefónico possibilitou-nos avaliar remotamente os RN, avaliar o cumprimento dos rastreios neonatais e do PNSIJ, identificar RN com critérios para observação urgente, RN sintomáticos ou preocupações parentais e - na ausência de sinais de alarme - auxiliar os pais na gestão no domicílio. Permitiu-nos ainda facultar às famílias um contacto de apoio num momento de maior vulnerabilidade e em que o acesso aos CSP se mostrou mais reduzido.

Dos RN, $40 \%$ não teve consulta médica no período neonatal, comprometendo o seguimento previsto. Menos de um em cada cinco RN foi observado nas primeiras duas semanas de vida, como preconiza o PNSIJ. A articulação entre a equipa hospitalar e os CSP de modo a assegurar a avaliação adequada não foi sempre a mais adequada. A vigilância do RN saudável é responsabilidade dos CSP e nestas circunstâncias foi comprometida. Podemos, a nível de cada hospital e respetivo agrupamento de centros de saúde, apurar os motivos pelos quais as primeiras consultas não foram marcadas. No que concerne a diminuição global da atividade assistencial, devemos reforçar que as consultas de Saúde Infantil dos primeiros dois anos de vida foram consideradas atividade assistencial essencial, pelo que devem ser priorizadas na reestruturação da atividade de cada centro de saúde. Já em relação à avaliação do RN 
em isolamento, em unidades sem condições logísticas para o fazer, temos de coordenar recursos humanos e equipamento para o fazer em visitas domiciliárias; podemos em conjunto definir as limitações de recursos que condicionaram esta falha no seguimento para as ultrapassar, considerando como opção a partilha de recursos (humanos e/ou materiais).

O alojamento conjunto possibilitou que a taxa de aleitamento materno (exclusivo ou misto) fosse elevada (97\%), contrariamente ao expectável com a separação mãe-filho. Curiosamente, a taxa de RN sob aleitamento materno exclusivo era discretamente superior no fim do período neonatal do que no momento da alta (56 vs $52 \%$ ), resultante do esforço de algumas mães para transitar de aleitamento misto para materno exclusivo. O aleitamento materno pode ter um efeito protetor, contribuindo para a ausência de infeção neonatal sintomática nesta amostra, com elevada taxa de aleitamento materno.

\section{4) RN com COVID-19}

Houve uma transmissibilidade mãe-filho de $5 \%$ nas primeiras 48 horas de vida, concordante com dados de outros estudos (1\% a 5\%). ${ }^{10,13,21,29}$ A nível nacional, em abril e maio, apesar de em $61 \%$ dos casos ter sido feita a separação mãe-filho, a transmissibilidade foi semelhante (4.7\%). ${ }^{21}$ A nível internacional, a evidência é contraditória: de acordo com a revisão sistemática publicada na Nature Communications, o alojamento conjunto aumenta o risco de transmissão mãe-filho ${ }^{12}$; já de acordo com a AAP, a transmissão mãe-filho é semelhante entre RN separados da mãe à nascença e RN mantidos em alojamento conjunto. ${ }^{10}$

Neste estudo não investigámos a hipótese de transmissão vertical (descrita em até $30 \%$ casos), ${ }^{12}$ através de estudo placentário ou serologia do RN.

Contrariamente a alguns dados publicados, ${ }^{9,12,27}$ em que até $55 \%$ dos RN com COVID-19 são sintomáticos, cerca de $25 \%$ são admitidos em UCIN e até $16 \%$ desenvolvem doença grave, neste estudo houve apenas um caso (25\%) de infeção sintomática, não houve casos de infeção neonatal grave nem de admissão na UCIN.

Apesar do alojamento conjunto poder aumentar risco do RN desenvolver COVID-19 após as 72 horas, não parece aumentar o risco de doença grave ${ }^{12}$; de facto, nesta pequena amostra de quatro RN com COVID-19 não houve doença grave. Para confirmar o aumento do risco após as 72 horas teríamos de testar o RN nesse período - tendo em conta que cerca de $90 \%$ teve alta até ao fim do terceiro dia de vida, implicaria prolongar o internamento ou realizar a testagem em ambulatório.

\section{5) Questões em aberto}

Com evidência cada vez mais sólida de que a infeção neonatal por SARS-CoV-2 é maioritariamente benigna, há entidades que questionam a necessidade de rastrear os RN de mãe com COVID-19 assintomáticos ${ }^{17}$ face ao reduzido impacto prático dessa testagem.

Apesar de questionável, a pesquisa de SARS-CoV-2 sistemática nestas circunstâncias permitiu a construção de evidência científica sobre a transmissão materna e a infeção neonatal. Assim, e como eventuais efeitos da infeção neonatal a médio/longo-prazo ainda são desconhecidos, consideramos lícito manter a abordagem atual.

A manter-se a testagem do $\mathrm{RN}$ assintomático, deverá ser escolhido melhor método e o momento ideal para a aplicar, em função do propósito para essa avaliação. Para rastreio e diagnóstico, o método mais fiável é a pesquisa de SARS-CoV-2 por RT-PCR, de maior sensibilidade entre o terceiro e o quinto dias de vida ${ }^{17,26}$; para confirmação diagnóstica em casos sintomáticos, ou para avaliação célere (por exemplo em contexto pré-operatório), justifica-se a pesquisa de antigénio de SARS-CoV-2, menos sensível do que a pesquisa por RT-PCR; para investigação de transmissão vertical, estão indicados o estudo anátomo-patológico da placenta e a serologia do RN.

Em relação ao seguimento após a alta hospitalar, está por definir a vigilância de forma mais adequada - clínica e epidemiológica.

Em relação à vigilância epidemiológica, a ausência de protocolo de cura dirigido ao RN com COVID-19 tornou inconsequentes as notificações no SINAVE e as próprias pesquisas de SARS-CoV-2 no RN, na medida em que não deram origem a um seguimento de Saúde Pública.

Para a vigilância clínica, é fundamental estruturar o seguimento destes RN de modo a cumprir o PNSIJ. As medidas criadas poderão manter a responsabilização dos CSP, como sucede com a população geral, ou aumentar a responsabilização da equipa hospitalar até à cura da mãe e/ou do RN. Poderá ainda ser planeada uma atitude articulada, por exemplo com 'vigilância domiciliária assegurada pelos CSP, com apoio da equipa de Neonatologia', proposta na Norma de Orientação da DGS. ${ }^{26}$

\section{CONCLUSÃO}

A COVID-19 em idade pediátrica, e em particular no período neonatal, tem baixa incidência e manifesta-se maioritariamente como infeção assintomática ou doença ligeira. A transmissão precoce de mãe para filho é incomum, com valores próximos de $5 \%$.

Dos resultados iniciais deste estudo, limitados pela dimensão da população (77 díades mãe/RN) e pelos seguimentos perdidos, destacam-se a baixa transmissibilidade de mãe para filho em alojamento conjunto com medidas de prevenção de transmissão, a ausência de doença neonatal grave, a incidência de prematuridade sobreponível à da população geral e a segurança do alojamento conjunto e da amamentação. Nesta pequena amostra, a abordagem adotada permitiu manter os benefícios do alojamento conjunto e da amamentação sem aumento da infeção neonatal sintomática.

A necessidade de testagem do $\mathrm{RN}$ assintomático é questionável, sobretudo pela ausência de implicação prática. São ainda incertos o método e o momento ideais para testar RN.

Face às falhas na vigilância clínica e epidemiológica, 
são necessárias medidas para melhorar a articulação com os CSP e a Saúde Pública.

\section{CONTRIBUTO DOS AUTORES}

IB: Desenho do estudo, recolha, armazenamento e análise de dados, revisão e discussão dos resultados.

RS: Recolha, armazenamento e análise de dados, revisão e discussão dos resultados.

BS: Desenho e coordenação do estudo, recolha de dados, revisão e discussão dos resultados.

JF: Desenho, revisão e discussão dos resultados.

SM: Desenho do estudo e recolha de dados.

AC: Desenho e coordenação, recolha de dados, revisão e discussão dos resultados.

\section{AGRADECIMENTOS}

Agradecemos os contributos dos Serviços de Obstetrícia e Ginecologia e de Otorrinolaringologia do Hospital Garcia de Orta.

\section{REFERÊNCIAS}

1. World Health Organization. Pneumonia of unknown cause - China. [acedido 2021 jan 15]. Disponível em: https://www.who.int/csr/don/05january-2020-pneumonia-of-unkown-cause-china/en/.

2. World Health Organization. WHO Director-General's opening remarks at the media briefing on COVID-19 - 11 March 2020. [acedido 2021 jan 15]. Disponivel em: https://www.who.int/director-general/speeches/ detail/who-director-general-s-opening-remarks-at-the-media-briefingon-covid-19---11-march-2020.

3. Oran D, Topol E. The proportion of SARS-CoV-2 infections that are asymptomatic: a systematic review. Ann Intern Med. 2021;M20-6976.

4. Wu Z, McGoogan J. Characteristics of and important lessons from the coronavirus disease 2019 (COVID-19) outbreak in China. JAMA. 2020;323:1239-42.

5. Lu X, Zhang L, Du H, Zhang J, Li Y, Qu J, et al. SARS-CoV-2 infection in children. N Engl J Med. 2020;382:1663-5.

6. Zimmermann P, Curtis N. Coronavirus infections in children including COVID-19. Pediatr Infect Dis J. 2020;39:355-68.

7. Viner R, Mytton O, Bonell C, Melendez-Torres G, Ward J, Hudson L, et al. Susceptibility to SARS-CoV-2 infection among children and adolescents compared with adults: a systematic review and meta-analysis. JAMA Pediatr. 2021;175:143-56.

8. Direção-Geral da Saúde. COVID-19 - Relatório de situação-01/01/2021. [acedido 2021 jan 16]. Disponível em: https://covid19.min-saude.pt/wpcontent/uploads/2021/01/305_DGS_boletim_20210101.pdf.

9. Trevisanuto D, Cavallin F, Cavicchiolo M, Borellini M, Calgaro S, Baraldi E. Coronavirus infection in neonates: a systematic review. Arch Dis Child Fetal Neonatal Ed. 2021;106:330-5.

10. American Academy of Pediatrics. Management of infants born to mothers with suspected or confirmed COVID-19. 2020. [acedido 2021 jan 16]. Disponível em: https://services.aap.org/en/pages/2019-novelcoronavirus-covid-19-infections/clinical-guidance/faqs-management-ofinfants-born-to-covid-19-mothers/.

11. Sutton D, Fuchs K, D'Alton M, Goffman D. Universal screening for SARSCoV-2 in women admitted for delivery. N Engl J Med. 2020;382:2163-4.

12. Raschetti R, Vivanti AJ, Vauloup-Fellous C, Loi B, Benachi A, De Luca D. Synthesis and systematic review of reported neonatal SARS-CoV-2 infections. Nat Commun. 2020;11:5164.

13. Ronchi A, Pietrasanta C, Zavattoni M, Saruggia M, Schena F, Sinelli M, et al. Evaluation of rooming-in practice for neonates born to mothers with severe acute respiratory syndrome coronavirus 2 infection in Italy. JAMA Pediatr. 2021;175:260-6.

14. Wang L, Shi Y, Xiao T, Fu J, Feng X, Mu D, et al. Chinese expert consensus on the perinatal and neonatal management for the prevention and control of the 2019 novel coronavirus infection (First edition). Ann Transl Med. 2020;8:47

15. Davanzo R, Moro G, Sandri F, Agosti M, Moretti C, Mosca F. Breastfeeding and coronavirus disease-2019: ad interim indications of

\section{PROTEÇÃO DE PESSOAS E ANIMAIS}

Os autores declaram que os procedimentos seguidos estavam de acordo com os regulamentos estabelecidos pelos responsáveis da Comissão de Investigação Clínica e Ética e de acordo com a Declaração de Helsínquia da Associação Médica Mundial atualizada em 2013.

\section{CONFIDENCIALIDADE DOS DADOS}

Os autores declaram ter seguido os protocolos do seu centro de trabalho acerca da publicação de dados.

\section{CONFLITOS DE INTERESSE}

Os autores declaram não ter conflitos de interesses relacionados com o presente trabalho.

\section{FONTES DE FINANCIAMENTO}

Este trabalho não recebeu qualquer tipo de suporte financeiro de nenhuma entidade no domínio público ou privado

the Italian Society of Neonatology endorsed by the Union of European Neonatal \& Perinatal Societies. Matern Child Nutr. 2020;16:e13010.

16. Sociedade Portuguesa de Neonatologia. Recomendações para a abordagem do recém-nascido em contacto com a infeção por SARS-CoV-2 (COVID-19). 2020. [acedido 2020 abr 04]. Disponível em: https://www.spneonatologia.pt/wp-content/uploads/2020/03/ COVID-19_170320.pdf.

17. British Association of Perinatal Medicine. BAPM - COVID-19: frequently asked questions within neonatal services. 2021. [acedido 2021 jan 16]. Disponível em: rcpch.ac.uk/resources/bapm-covid-19-frequently-askedquestions-within-neonatal-services.

18. Direção-Geral da Saúde. Programa Nacional de Saúde Infantil e Juvenil. Norma da Direção-Geral da Saúde. 2013 [acedido 2021 jan 16]. Disponível em: dgs.pt/directrizes-da-dgs/normas-e-circularesnormativas/norma-n-0102013-de-31052013-jpg.aspx.

19. Campos A, Leça A, Campos D, Lança F, Almeida M, Moucho M, et al. COVID-19: gravidez e parto. Orientação. Direção Geral da Saúde; 2020 [acedido 2021 jan 16]. Disponível em: https://www.dgs.pt/normasorientacoes-e-informacoes/orientacoes-e-circulares-informativas/ orientacao-n-0182020-de-30032020-pdf.aspx.

20. Orfão A, Santos A, Gouveia C, Santos C. Registo do aleitamento materno | Relatório janeiro a dezembro 2013. Direção Geral da Saúde; 2014 [acedido 2021 mar 13]. Disponível em: https://www.dgs.pt/ documentos-e-publicacoes/iv-relatorio-com-os-dados-do-registo-doaleitamento-materno-2013-pdf.aspx.

21. Costa M, Vala B, Aquino J, Bolhota-Xavier J. Pandemia COVID-19: atitudes dos serviços de Neonatologia. Primeiras Jornadas Digitais de Pediatria; 2020 [acedido 2021 jan 16]. Disponível em: https://spp. eventkey.pt/reports/reports.aspx?ref=resumofinal 1 \&evento=11\&formula rio $=33 \&$ render $=$ pagina \&cod $=10223 \&$ chave $=004282 \mathrm{~A} 616$.

22. Ballestero $C$. "Nunca mais vi o Jaime, nem tive contacto desde o dia em que nasceu". A história de uma mãe com COVID-19. MAGG; 2020 [acedido 2021 jan 16]. Disponível em: https://magg.sapo.pt/saude/ artigos/nunca-mais-vi-o-jaime-nem-tive-contacto-desde-o-dia-em-quenasceu-a-historia-de-uma-mae-com-covid-19.

23. Ribeiro C. Depois de 13 testes à covid-19, Eunice pôde beijar o seu bebé pela primeira vez. Público; 2020 [acedido 2021 jan 16]. Disponível em: https://www.publico.pt/2020/05/27/impar/noticia/13-testes-covid19eunice-beijar-bebe-primeira-1918377.

24. Correia A. Bebé filha de mãe com Covid-19 "está bem" e à guarda do hospital São João. Jornal de Notícias; 2020 [acedido 2021 jan 16] Disponível em: https://www.jn.pt/nacional/bebe-filga-de-mae-conmcovid-esta-bem-e-a-guarda-do-hospital-sao-joao-11959246.html.

25. Stuebe A. Should infants be separated from mothers with COVID-19? First, do no harm. Breastfeed Med. 2020;15:351-2.

26. Mimoso G, Dinis A, Ventura T, Leça A. COVID-19: cuidados ao recémnascido na maternidade. Orientação. Direção Geral da Saúde; 2020 
[acedido 2021 jan 16]. Disponível em: https://www.dgs.pt/directrizes-dadgs/orientacoes-e-circulares-informativas/orientacao-n-0262020-de19052020-pdf.aspx.

27. Allotey J, Stallings E, Bonet M, Yap M, Chatterjee S, Kew T, et al. Clinical manifestations, risk factors, and maternal and perinatal outcomes of coronavirus disease 2019 in pregnancy: living systematic review and meta-analysis. BMJ. 2020;370:m3320.
28. Gale C, Quigley M, Placzek A, Knight M, Ladhani S, Draper E, et al. Characteristics and outcomes of neonatal SARS-CoV-2 infection in the UK: a prospective national cohort study using active surveillance. Lancet Child Adolesc Heal. 2021;5:113-21.

29. Di Toro F, Gjoka M, Di Lorenzo G, De Santo D, De Seta F, Maso G, et al. Impact of COVID-19 on maternal and neonatal outcomes: a systematic review and meta-analysis. Clin Microbiol Infect. 2021;27:36-46. 